\title{
DUST AROUND HERBIG AE/BE STARS
}

\author{
KYUNG-WON SUH \\ Department of Astronomy and Space Science, Chungbuk National University, Cheongju 361-763, Korea \\ E-mail : kwsuh@chungbuk.ac.kr
}

(Received October 20, 2010; Revised November 19, 2010; Accepted December 08, 2010)

\begin{abstract}
We model dust around Herbig Ae/Be stars using a radiative transfer model for multiple isothermal circumstellar dust shells to reproduce the multiple broad peaks in their spectral energy distributions (SEDs). Using the opacity functions for various types of dust grains at different temperatures, we calculate the radiative transfer model SEDs for multiple dust shells. For eight sample stars, we compare the model results with the observed SEDs including the Infrared Space Observatory (ISO) and $A K A R I$ data. We present model parameters for the best fit model SEDs that would be helpful to understand the overall structure of dust envelopes around Herbig Ae/Be stars. We find that at least four separate dust components are required to reproduce the observed SEDs. For all the sample stars, two innermost dust components (a hot component of 1000-1500 K and a warm component of 300-600 K) with amorphous silicate and carbon grains are needed. Crystalline dust grains (corundum, forsterite, olivine, and water ice) are needed for some objects. Some crystalline dust grains exist in cold regions as well as in hot inner shells.
\end{abstract}

Key words : stars: pre-main sequences — infrared: stars — circumstellar matter — dust: extinction - radiative transfer

\section{INTRODUCTION}

Herbig Ae/Be (HAEBE) stars are generally believed to be intermediate-mass $\left(M \sim 2-10 \quad M_{\odot}\right)$ pre-mainsequence (PMS) stars. As a consequence of the star formation process they are surrounded by a gas and dust envelope and/or disk (e.g., Waters \& Waelkens 1998).

To understand the physical and chemical properties of the envelope or the disk, high resolution spectral observations in IR are required. The infrared space observatory (ISO) and Spitzer missions provided the essential data. Some HAEBE stars show prominent features of various dust species including crystalline grains (e.g., Meeus et al. 2001; Juhász et al. 2010).

The infrared SEDs of the HAEBE stars show multiple broad peaks at 5 - $200 \mu \mathrm{m}$ (e.g., Suh, Kim, \& Baek 2002). A reasonable explanation for this would be that there are multiple dust components radiating multiple regions of infrared wavelengths.

In this paper, we use a radiative transfer model for multiple isothermal circumstellar dust shells (Towers \& Robinson 2009) to consider multiple components of dust. We model the dust envelopes around HAEBE stars using optical properties of various amorphous and crystalline dust grains at different temperatures.

We compare the model results with the observed SEDs of the stars including the ground-based, IRAS, $I S O$ and $A K A R I$ data. Using the comparison, we determine the model parameters for the best fit model
SEDs that would be helpful to understand the overall structure of dust envelopes around HAEBE stars.

\section{SAMPLE STARS}

In this paper, we choose eight HAEBE stars divided into three groups (see section 4 for the explanations about the groups). For these stars, good quality observational data including the $I S O$ spectra in wide wavelength ranges are available. The sample stars are listed in Table 1. For each object, the table lists the $I R A S$ point source catalogue (PSC) number, the number of the $I S O$ data used for this paper, the $A K A R I$ infrared camera (IRC) PSC number, the $A K A R I$ far-infrared surveyor (FIS) bright source catalogue (BSC) number, the effective temperature, the distance, and the luminosity.

For the $I S O$ data, we use the $I S O$ short wavelength spectrometer (SWS; $\lambda=2.4-45.2 \mu \mathrm{m}$ ) and long wavelength spectrometer (LWS; $\lambda=43-197 \mu \mathrm{m}$ ) data reduced by Meeus et al. (2001).

The $A K A R I$ made an all-sky survey with the IRC and FIS. We use the $A K A R I$ PSC data at two bands (9 and $18 \mu \mathrm{m}$ ) obtained by IRC and the BSC data at four bands $(65,90,140$ and $160 \mu \mathrm{m})$ obtained by FIS. For each object, we have cross-identified the $A K A R I$ source by finding the nearest one from the position information.

To obtain the standard flux in $W / m^{2}$ for all the data, we use the zero-magnitude calibrating method. 
Table 1.

Sample HAEBE stars.

\begin{tabular}{|c|c|c|c|c|c|c|c|c|}
\hline Group $^{a}$ & Name & $I R A S$ PSC & $\begin{array}{l}\text { ISO datab } \\
\text { (SWS;LWS) }\end{array}$ & $A K A R I$ IRC & $A K A R I$ FIS & $\begin{array}{c}T_{e f f}^{a} \\
(\mathrm{~K})\end{array}$ & $\begin{array}{c}d^{a} \\
(p c)\end{array}$ & $\begin{array}{l}L_{*}{ }^{a} \\
\left(L_{\odot}\right)\end{array}$ \\
\hline \multirow[t]{2}{*}{$\mathrm{A}$} & HD 100453 & $11307-5402$ & $(1 ;-)$ & $1133055-541928$ & $1133053-541929$ & $7500^{1}$ & - & - \\
\hline & AB Aur & $04525+3028$ & $(1 ;-)$ & $0455458+303303$ & $0455460+303320$ & $9750^{1}$ & $144^{1}$ & $52.48^{2}$ \\
\hline \multirow[t]{2}{*}{ B } & HD 144432 & $16038-2735$ & $(1 ;-)$ & $1606579-274310$ & $1606579-274308$ & $8000^{1}$ & $200^{2}$ & $30.20^{2}$ \\
\hline & HD 163296 & $17533-2156$ & $(1 ;-)$ & $1640179-235346$ & $1640176-235344$ & $10500^{1}$ & $122^{1}$ & $30.20^{2}$ \\
\hline $\mathrm{C}$ & HD 100546 & $11312-6955$ & $(1 ; 1)$ & $1133254-701140$ & $1133251-701146$ & $11000^{1}$ & $103^{1}$ & $32.36^{2}$ \\
\hline
\end{tabular}

${ }^{a}$ : see section 4 for the explanations; ${ }^{b} 1$ : from Meeus et al. 2001, 2: from van den Ancker 1997, 3: from Hillenbrand et al. 1992.

The zero-magnitude calibrating data are taken from the related references. The observed SEDs of the eight stars are displayed in Fig. 2 through 4. For IRAS and $A K A R I$ catalogue data, we use only good quality data.

\section{DUST ENVELOPE MODEL CALCULA- TIONS}

The infrared SEDs of the HAEBE stars show multiple broad peaks at $5-200 \mu \mathrm{m}$ (e.g., Suh et al. 2002). A reasonable explanation for this would be that there are multiple dust components radiating multiple regions of infrared wavelengths. It is difficult to reproduce the observed SEDs with a single component dust shell model (e.g., DUSTY code developed by Ivezić \& Elitzur 1997). Many investigators (e.g., Miroshnichenko et al. 1999; Whitney et al. 2003) tried to model the multiple components (shells, spheroids, or disks) of the dust envelopes around HAEBE stars with various methods and degrees of sophistication.

The radiative transfer model for multiple isothermal spherically symmetric circumstellar dust shells developed by Towers \& Robinson (2009) assumes that each dust shell is in local thermodynamical equilibrium (LTE) and that the temperature is constant. Dust grains in the innermost shell absorb the radiation from the central star and radiate at the equilibrium temperature. Dust grains in an outer shell absorb the radiation from the central star and the inner shell(s) and radiate at the equilibrium temperature. The scattering of light is ignored. These assumptions would be reasonable approximations for studying dust around HAEBE stars.

In this paper, we use the radiative transfer model for multiple isothermal circumstellar dust shells (Towers \& Robinson 2009) to consider multiple components of the dust envelope. Though it is a relatively simple model, it can use flexible parameters of dust properties to treat radiative processes through centrally heated multiple dust shells. The code developed by Towers \& Robin- son (2009) would be useful to investigate the overall properties of complicated distribution of dust around a central star. We model dust envelopes around HAEBE stars using optical properties of various amorphous and crystalline dust grains at different temperatures.

For the central star, we assume simple blackbody radiation. For each object, we use the luminosity and effective temperature obtained by previous authors as listed in Table 1. For HD 100453, we use $L=50 L_{\odot}$.

\subsection{Dust Opacity}

The best fit model for the observed SED requires a proper combination of multiple isothermal components with different sets of various dust opacity functions. We have tried to use as many dust species as possible. We find that seven dust species (amorphous silicate, amorphous carbon, amorphous $\mathrm{Mg}_{0.6} \mathrm{Fe}_{0.4} \mathrm{O}$, crystalline corundum, crystalline olivine, crystalline forsterite, and crystalline water ice) are necessary to reproduce the SEDs of sample HAEBE stars.

In this paper, we do not consider PAH (Polycyclic Aromatic Hydrocarbon) for the radiative transfer model calculations because their thermal properties are not well known yet.

For amorphous silicate, we use the optical constants derived by Suh (1999) for warm silicate. For amorphous carbon (AMC), we use the optical constants derived by Suh (2000). For amorphous $\mathrm{Mg}_{0.6} \mathrm{Fe}_{0.4} \mathrm{O}$, we use the optical constants obtained by Henning et al. (1995). For crystalline $\mathrm{H}_{2} \mathrm{O}$ ice, we use the optical constants obtained by Bertie (1969). For crystalline olivine, we use the optical constants obtained for spherical crystalline olivine grains $\left(\mathrm{Mg}_{1.9} \mathrm{Fe}_{0.1} \mathrm{SiO}_{4}\right)$ obtained by Fabian et al. (2001). For all the five species, the extinction efficiency factors are calculated for spherical dust grains (Bohren \& Huffman 1983) from the optical constants given in the references. The radii of the spherical dust grains are assumed to be 0.1 $\mu \mathrm{m}$ uniformly.

For crystalline corundum, we use the extinction data 


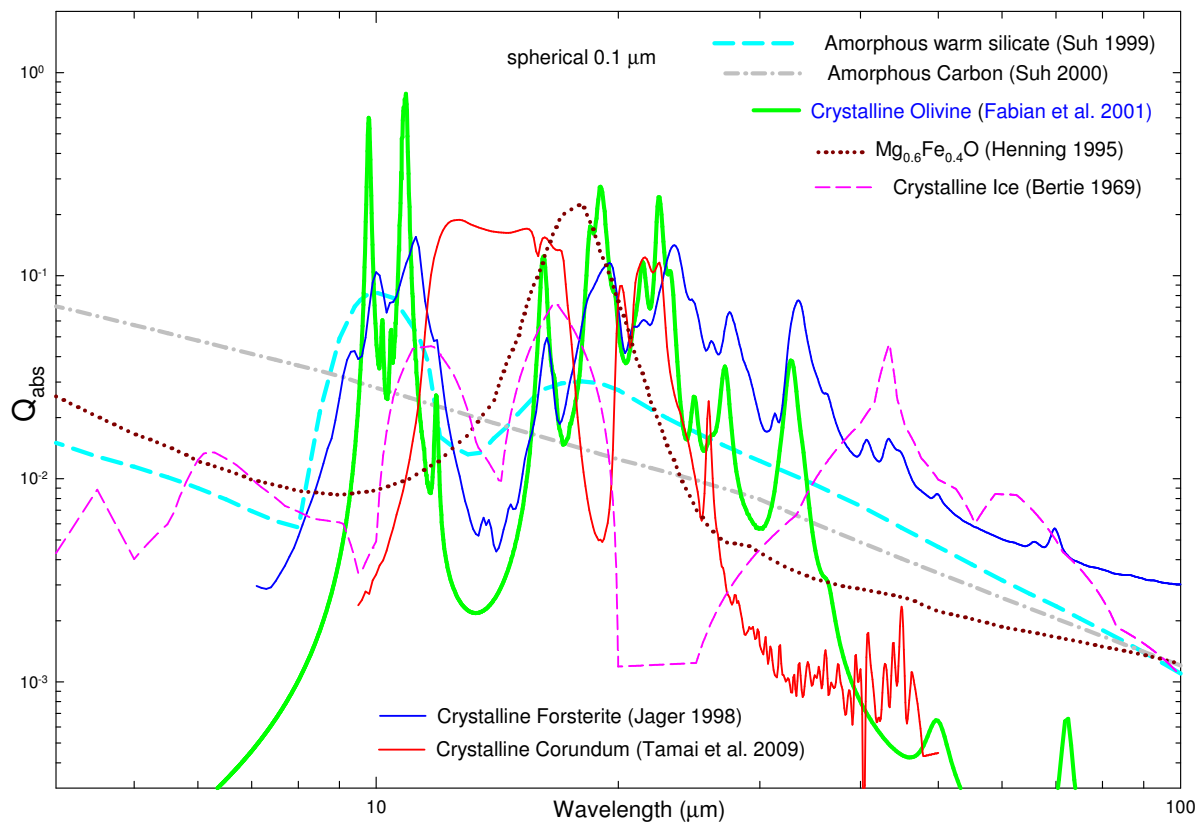

Fig. 1.- Dust opacity functions for the seven species.

Table 2.

The model parameters of the multiple isothermal dust shells for the best fit model SEDs

\begin{tabular}{|c|c|c|c|c|c|c|}
\hline Name & $\begin{array}{l}\text { Central star } \\
T_{B B}(\mathrm{~K})\end{array}$ & $L_{*}\left(L_{\odot}\right)$ & $\begin{array}{l}\text { 1st Shell } \\
\mathrm{T}(\mathrm{K}), \tau_{10}, r\left(R_{*}\right)\end{array}$ & Opacity $^{1}$ & $\begin{array}{l}\text { 2nd Shell } \\
\mathrm{T}(\mathrm{K}), \tau_{10}, r\left(R_{*}\right)\end{array}$ & Opacity $^{1}$ \\
\hline HD 100453 & 7500 & 50 & $1000,3.0 \mathrm{E}-3,1.7 \mathrm{E} 2$ & $0.95 \mathrm{a}+0.05 \mathrm{~s}$ & $300,8.0 \mathrm{E}-4,3.2 \mathrm{E} 3$ & $0.95 \mathrm{a}+0.05 \mathrm{~s}$ \\
\hline HD 104237 & 10500 & 60 & $1300,2.0 \mathrm{E}-3,2.1 \mathrm{E} 2$ & $0.5 \mathrm{a}+0.5 \mathrm{~s}$ & $500,1.0 \mathrm{E}-3,2.0 \mathrm{E} 3$ & $0.5 \mathrm{a}+0.5 \mathrm{~s}$ \\
\hline HD 144432 & 8000 & 30 & $1200,4.0 \mathrm{E}-3,1.3 \mathrm{E} 2$ & $0.3 \mathrm{a}+0.6 \mathrm{~s}+0.1 \mathrm{c}$ & $500,4.5 \mathrm{E}-3,9.1 \mathrm{E} 2$ & $0.3 \mathrm{a}+0.6 \mathrm{~s}+0.1 \mathrm{C}$ \\
\hline HD 163296 & 10500 & 30 & $1200,3.0 \mathrm{E}-3,2.5 \mathrm{E} 2$ & $0.6 \mathrm{a}+0.4 \mathrm{~s}$ & $500,2.0 \mathrm{E}-3,1.9 \mathrm{E} 3$ & $0.6 \mathrm{a}+0.4 \mathrm{~s}$ \\
\hline HD 150193 & 10000 & 27 & $1000,4.0 \mathrm{E}-3,3.4 \mathrm{E} 2$ & $0.5 \mathrm{a}+0.4 \mathrm{~s}+0.1 \mathrm{C}$ & $600,8.0 \mathrm{E}-4,1.1 \mathrm{E} 3$ & $0.5 \mathrm{a}+0.4 \mathrm{~s}+0.1 \mathrm{C}$ \\
\hline HD 142527 & 6250 & 69 & $1200,8.0 \mathrm{E}-3,6.8 \mathrm{E} 1$ & $0.5 \mathrm{a}+0.5 \mathrm{~s}$ & $500,5.5 \mathrm{E}-3,4.8 \mathrm{E} 2$ & \\
\hline HD 100546 & 11000 & 32 & $1500,3.0 \mathrm{E}-3,1.7 \mathrm{E} 2$ & $0.3 \mathrm{a}+0.6 \mathrm{~s}+0.1 \mathbf{V}$ & $500,1.0 \mathrm{E}-3,2.2 \mathrm{E} 3$ & $0.3 \mathrm{a}+0.6 \mathrm{~s}+0.1 \mathbf{V}$ \\
\hline \multirow[t]{2}{*}{ Name } & 3rd Shell & & 4th Shell & & 5th Shell & \\
\hline & $\mathrm{T}(\mathrm{K}), \tau_{10}, r\left(R_{*}\right)$ & Opacity $^{1}$ & $\mathrm{~T}(\mathrm{~K}), \tau_{10}, r\left(R_{*}\right)$ & Opacity $^{1}$ & $\mathrm{~T}(\mathrm{~K}), \tau_{10}, r\left(R_{*}\right)$ & Opacity $^{1}$ \\
\hline HD 100453 & $110,3.0 \mathrm{E}-3,4.1 \mathrm{E} 4$ & $1 \mathrm{a}$ & $45,1.2 \mathrm{E}-3,4.4 \mathrm{E} 5$ & $1 \mathrm{a}$ & - & - \\
\hline HD 104237 & $150,4.0 \mathrm{E}-4,3.3 \mathrm{E} 4$ & $0.5 \mathrm{a}+0.5 \mathrm{~s}$ & $60,1.0 \mathrm{E}-4,5.3 \mathrm{E} 5$ & $1 \mathrm{a}$ & - & - \\
\hline AB Aur & $150,2.5 \mathrm{E}-3,2.4 \mathrm{E} 4$ & $0.7 \mathrm{a}+0.3 \mathrm{~s}$ & $50,2.5 \mathrm{E}-3,5.9 \mathrm{E} 5$ & $1 \mathrm{a}$ & - & - \\
\hline HD 144432 & $120,2.5 \mathrm{E}-3,2.5 \mathrm{E} 4$ & $0.3 \mathrm{a}+0.7 \mathrm{~s}$ & $60,3.0 \mathrm{E}-4,2.6 \mathrm{E} 5$ & $1 \mathrm{a}$ & - & - \\
\hline HD 163296 & $200,1.0 \mathrm{E}-3,1.6 \mathrm{E} 4$ & $0.6 \mathrm{a}+0.4 \mathrm{~s}$ & $100,5.0 \mathrm{E}-4,9.7 \mathrm{E} 4$ & $0.6 \mathrm{a}+0.4 \mathrm{~s}$ & $27,5.0 \mathrm{E}-4,4.4 \mathrm{E} 6$ & $1 \mathrm{a}$ \\
\hline HD 150193 & $250,2.0 \mathrm{E}-3,7.8 \mathrm{E} 3$ & $0.5 \mathrm{a}+0.5 \mathrm{~s}$ & $100,8.0 \mathrm{E}-4,8.0 \mathrm{E} 4$ & $0.5 \mathrm{a}+0.5 \mathrm{~s}$ & $60,1.5 \mathrm{E}-4,4.4 \mathrm{E} 5$ & $1 \mathrm{a}$ \\
\hline
\end{tabular}

1 a: amorphous carbon (Suh 2000), s: amorphous silicate (Suh 1999), m: amorphous $\mathrm{Mg}_{0.6} \mathrm{Fe}_{0.4} \mathrm{O}$ (Henning et al. 1995 ), C: crystalline corundum (Tamanai et al. 2009), F: crystalline forsterite (Jäger et al. 1998), V: crystalline olivine (Fabian et al. 2001), I: crystalline $\mathrm{H}_{2} \mathrm{O}$ ice (Bertie 1969), $\mathbf{X}=0.4 \mathrm{a}+0.45 \mathrm{~s}+0.05 \mathbf{V}+0.1 \mathbf{C}, \mathbf{Y}=0.4 \mathrm{a}+0.52 \mathrm{~m}+0.04 \mathbf{V}+0.04 \mathbf{I}, \mathbf{Z}=0.4 \mathrm{a}+0.45 \mathrm{~s}+0.1 \mathbf{F}+0.05 \mathbf{C}$ 
of $\alpha-\mathrm{Al}_{2} \mathrm{O}_{3}$ (corundum-sample 1 ) obtained by Tamanai et al. (2009). For crystalline forsterite, we use the the extinction data obtained by Jäger et al. (1998).

Dust opacity functions for the seven species are displayed in Fig. 1. Spherical crystalline olivine grains produce 27 and $33 \mu \mathrm{m}$ features and multiple peaks around $22 \mu \mathrm{m}$. The crystalline forsterite produce 19.5, $23.5,27.5$, and $33.5 \mu \mathrm{m}$ features.

\subsection{Model SEDs}

We have performed various radiative transfer model calculations in the wavelength range 0.01 to $36000 \mu \mathrm{m}$. We choose $10 \mu \mathrm{m}$ as the fiducial wavelength that sets the scale of the optical depth $\left(\tau_{10}\right)$. We have computed the model SEDs for various optical depths of the multiple dust shells with different dust opacity.

For each object, we have tried to find the best fit model SED for the observed one. Once we have a set of reasonable model parameters, we compare the model result with the observed SED and repeat revising the related parameter(s) until we get a satisfactory fit in the entire wavelength range. Considering the overall shape of the SED, we guess the initial parameters for the central star and the innermost dust shells. Then we try to use various dust temperatures and opacity functions in the outer shell(s) to reproduce the special features of the observed SED in the far-IR (FIR) region. However, we may have to revise the parameters for the multiple dust shells repeatedly because of the correlated absorption processes.

The model parameters of the multiple isothermal dust shells for the best fit model SEDs are listed in Table 2. For each object, the parameters of the central star (the blackbody temperature and luminosity) and those for multiple dust shells are listed. For each dust shell, the equilibrium temperature, the dust optical depth $\left(\tau_{10}\right)$, the radius of the shell $(r)$ in the unit of the radius of the central star $\left(R_{*}\right)$, and the dust opacity function are listed. All of the above parameters except the radius of the shell $(r)$ are input parameters. For each model, the code calculates the radii $(r)$ of the multiple dust shells and the model SED.

Fig. 2 through 4 show the best fit model SEDs compared with the observed SEDs for the eight sample stars. In next section, we will discuss the meaning of the model parameters for each object.

\section{SED COMPARISON}

For each object, we have tried to reproduce all the features and characteristics of the observed SEDs by the radiative transfer model in a wide wavelength range. However, we could not reproduce some of them because of the simplicity of the model we used and/or lack of general knowledge about some dust materials. We divide the sample stars into three groups according to the characteristics of the model parameters for the best fit SEDs.

\subsection{Group A}

For group A stars, we use four separate dust shells with two dust species (amorphous silicate and AMC). Generally, a cold (45-60 K) dust shell with featureless AMC grains reproduces the FIR region fairly well. Unlike other groups, group A stars do not show prominent crystalline dust features.

Though HD 100453 does not show silicate features, we find that a small portion (5\%) of silicate grains at hot $(1000 \mathrm{~K})$ and warm $(300 \mathrm{~K})$ shells improve the overall fit.

HD 104237 requires hot $(1300 \mathrm{~K})$ silicate $(50 \%)$ and AMC. We were not able to reproduce the peak at 30 $\mu \mathrm{m}$ with any other model parameters and known dust species.

AB Aur also requires hot $(1200 \mathrm{~K})$ silicate $(70 \%)$ and AMC. Though FeO dust grains in shapes of continuous distribution of ellipsoids (CDE) at $150 \mathrm{~K}$ may improve the fit at $23 \mu \mathrm{m}$, we were not able to improve the overall fit by adding the $\mathrm{FeO}$ grains.

\subsection{Group B}

For group B stars, we use four separate dust shells with three dust species or five separate dust shells with two to three dust species. For the two objects in the group, we use crystalline corundum as well as amorphous silicate and AMC (see Table 2). Again for this group, a cold (27-60 K) dust shell with featureless AMC grains reproduces the FIR region fairly well.

HD 144432 requires hot $(1200$ and $500 \mathrm{~K})$ silicate $(60 \%)$, crystalline corundum (10\%), and AMC. The crystalline corundum in the inner dust shells significantly improves the fit at $12-20 \mu \mathrm{m}$ region.

HD 163296 requires hot (1200 and $500 \mathrm{~K})$ silicate $(40 \%)$ and AMC. There is no sign of crystalline corundum. For this object, we use only two dust species (amorphous silicate and AMC) like all of group A stars.

HD 150193 requires hot $(1000$ and $600 \mathrm{~K})$ silicate (40\%), crystalline corundum (10\%), and AMC. Crystalline corundum in the hot shells improves the fit at $12-20 \mu \mathrm{m}$ region.

\subsection{Group C}

For group C stars, we use five separate dust shells with five to seven dust species including crystalline dust (corundum, olivine forsterite, and water ice) as well as amorphous dust (silicate, AMC, and $\mathrm{Mg}_{0.6} \mathrm{Fe}_{0.4} \mathrm{O}$ ).

For this group, a cold $(48-60 \mathrm{~K})$ dust shell of amorphous and crystalline silicate as well as featureless AMC grains reproduces the FIR region fairly well.

HD 142527 shows emission features around $10 \mu \mathrm{m}$ similar to crystalline olivine at $500 \mathrm{~K}$. Crystalline corundum at $500 \mathrm{~K}$ improves the fit at $15 \mu \mathrm{m}$. The conspicuous emission feature at $43 \mu \mathrm{m}$ looks to be due to cold $(48 \mathrm{~K})$ crystalline $\mathrm{H}_{2} \mathrm{O}$ ice. The cold dust component $(48 \mathrm{~K})$ with a mixture of $\mathrm{Mg}_{0.6} \mathrm{Fe}_{0.4} \mathrm{O}$ and crys- 

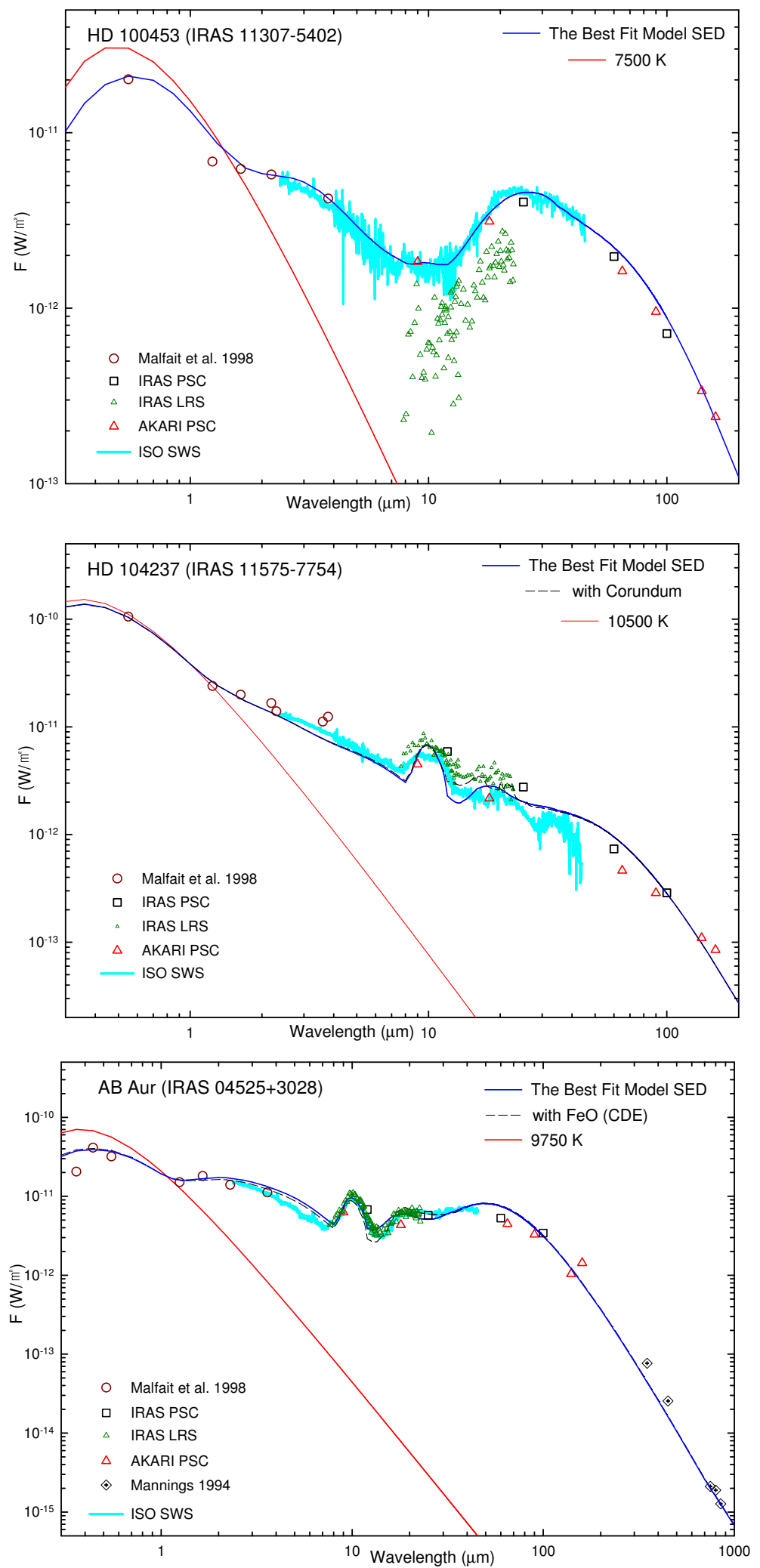

Fig. 2.- Observations compared with model SEDs for the group A stars. 

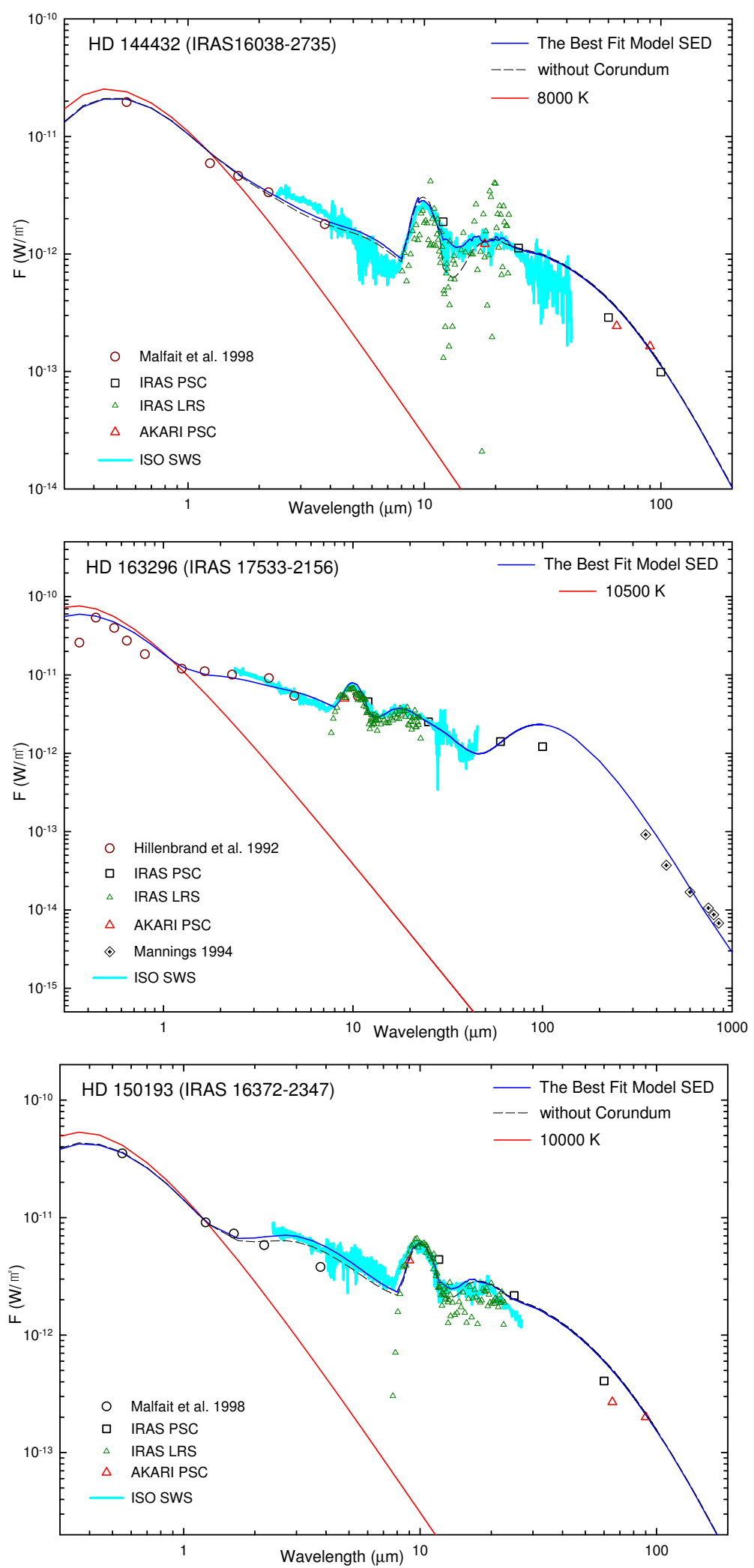

Fig. 3.- Observations compared with model SEDs for the group B stars. 

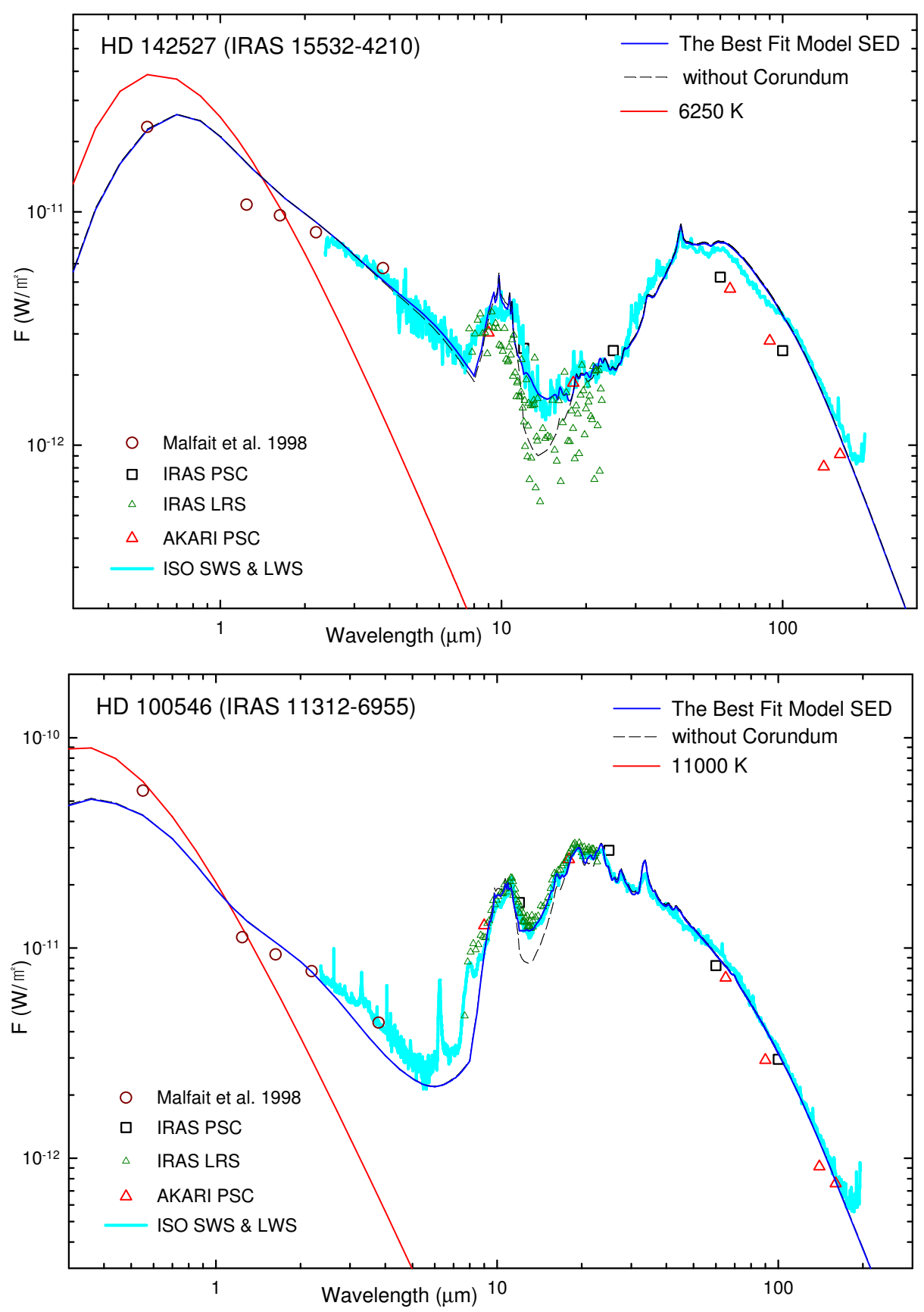

Fig. 4.- Observations compared with model SEDs for the group C stars. 
talline olivine improves the overall fit in the FIR region. Although the seven dust species make the best fit model SED, HD 142527 shows unknown emission features at 36 and $47 \mu \mathrm{m}$.

For HD 100546, the crystalline forsterite dust grains at 200,100 , and $60 \mathrm{~K}$ reproduce the observed fine features pretty well. They reproduce the similar 19.5, 23.5, 27.5, and $33.5 \mu \mathrm{m}$ features. The crystalline olivine grains at $500 \mathrm{~K}$ reproduce the multiple peaks around $20 \mu \mathrm{m}$. Crystalline corundum in a warm $(200 \mathrm{~K})$ shell improves the fit at $12-20 \mu \mathrm{m}$ region.

The observed SED of HD 100546 shows very prominent PAH emission features at 3.2, 6.2, 7.7, and $8.6 \mu \mathrm{m}$ which could not be reproduced with our model (see section 3.1). Brittain, Najita, \& Carr (2009) argued that the inner hole of HD 100546 is not simply a hole in the dust opacity but is likely cleared of gas as well. Efficient heating of the outer disk by the star HD 100546 would produce the prominent PAH features.

\subsection{Discussion on Crystalline Silicate}

Because crystalline silicate grains show very sharp features, even a small portion (about $5 \%$ ) can be easily detectable. It is quite evident that crystalline silicate grains (forsterite and olivine) exist in cold (48 $100 \mathrm{~K}$ ) outer dust shells (or disks) of many HAEBE stars (e.g., this paper; Juhász et al. 2010). Though crystalline silicates are abundant in many young stellar objects (YSOs) and solar system comets, they are essentially missing from the interstellar medium (ISM) (e.g., Juhász et al. 2010). It would be reasonable to assume that crystallization occurs in the low temperature envelopes (or disks) of YSOs.

Some known processes of crystallization (annealing and direct condensation from the gas phase) require high temperature (about $1000 \mathrm{~K}$ ) (Fabian et al. 2000). On the other hand, Carrez et al. (2002) and Kimura et al. (2008) suggested a mechanism of crystallization at low temperature by reporting that amorphous silicate grains were crystallized to forsterite by electron-beam irradiation. YSOs are known to undergo active and frequent flaring events in which electrons are accelerated. Therefore, the electron irradiation of dust in YSO environments could explain the origin of the crystalline silicate grains around HAEBE stars.

\section{CONCLUSIONS}

We have modeled dust around HAEBE stars using a radiative transfer model for multiple isothermal circumstellar dust shells to reproduce the multiple broad peaks in SEDs. By comparing the model results with the observed SEDs in a wide wavelength range for the eight sample stars, we have presented the model parameters of the best fit model SEDs that would be helpful to understand the overall structure of dust envelopes around HAEBE stars.

Because of the violent star forming environments (flaring events, accretion processes, etc.), the dust grains around a HAEBE star may have various physical and chemical properties. And there could be frequent changes in the geometrical distribution of the dust grains. Therefore, we may not be able to explain the complicated variety of dust for the eight sample stars (see Table 2) in a straightforward way. However, we may infer some important ideas about the nature of an object from the revealed information about the dust (see section 4).

We have found that at least four separate dust components are required to reproduce the observed SEDs. For all the sample stars, two innermost dust components (a hot component of 1000-1500 K and a warm component of 300-600 K) with amorphous silicate and AMC grains are needed. Crystalline dust grains (corundum, forsterite, olivine, and water ice) are needed for some objects.

Crystalline corundum grains have been found to be present in a half of the sample stars. Corundum looks to be one of the major dust components in YSOs. More investigations are necessary to find the contribution of crystalline and amorphous corundum grains to the SEDs for a larger sample of YSOs.

For the two stars in group $\mathrm{C}$, the crystalline silicate grains (forsterite and olivine) exist in cold (48 - 100 $\mathrm{K}$ ) outer dust shells as well as in hot inner dust shells. Though the reason for the existence of low temperature crystalline silicate is still uncertain, the electron irradiation of dust in YSO environments could be a possible scenario. More investigations would be able to reveal useful information about physical and chemical properties of dust around HAEBE stars and general environments of star forming regions.

\section{ACKNOWLEDGMENTS}

This work was supported by the research grant of the Chungbuk National University in 2009. This research is based on observations with AKARI, a JAXA project with the participation of ESA.

\section{REFERENCES}

Bertie, J. E. 1969, Absorptivity of Ice in the Range 4000-30 $\mathrm{cm}^{-1}$, The Journal of Chemical Physics, 50, 451

Bohren, C. F., \& Huffman, D. R. 1983, Absorption and Scattering of Light by Small Particles (New York: Wiley)

Brittain, S. D., Najita, J. R., \& Carr, J. S. 2009, Tracing the Inner Edge of the Disk Around HD 100546 with Rovibrational CO Emission Lines, ApJ, 702, 85

Carrez, P., Demyk, K., Leroux, H., Cordier, P., Jones, P., \& d'Hendecourt, L. 2002, Low-temperature Crystallization of $\mathrm{MgSiO}_{3}$ Glasses under Electron Irradiation: Possible Implications for Silicate Dust Evolution in Circumstellar Environments, Meteoritics Planet. Sci., 37, 1615 
Fabian, D., Jager, C., Henning, Th., Dorschner, J., \& Mutschke, H. 2000, Steps toward Interstellar Silicate Mineralogy. V. Thermal Evolution of Amorphous Magnesium Silicates and Silica, A\&A, 364, 282

Fabian, D., Henning, T., Jäger, C., Mutschke, H., Dorschner, J., \& Wehrhan, O. 2001, Steps toward Interstellar Silicate Mineralogy VI. Dependence of Crystalline Olivine IR Spectra on Iron Content and Particle Shape, A\&A, 378, 228

Henning, Th., Begemann, B., Mutschke, H., \& Dorschner, J. 1995, Optical Properties of Oxide Dust Grains, A\&AS, 112, 143

Hillenbrand, L. A., Strom, S. E., Vrba, F. J., \& Keene, J. 1992, Herbig Ae/Be Stars - Intermediate-Mass Stars Surrounded by Massive Circumstellar Accretion Disks, ApJ, 397, 613

Ivezić, A., \& Elitzur, M. 1997, Self-Similarity and Scaling Behaviour of Infrared Emission from Radiatively Heated Dust - I. Theory, MNRAS, 287, 799

Jäger, C., Molster, F. J., Dorschner, J., Henning, Th., Mutschke, H., \& Waters, L. B. F. M. 1998, Steps toward Interstellar Silicate Mineralogy IV. The Crystalline Revolution, A\&A, 339, 904

Juhász, A., Bouwman, J., Henning, Th., Acke, B., van den Ancker, M. E., Meeus, G., Dominik, C., Min, M., Tielens, A. G. G. M., \& Waters, L. B. F. M. 2010, Dust Evolution in Protoplanetary Disks Around Herbig Ae/Be Stars- the Spitzer View, ApJ, 721,431

Kimura, Y., Miyazaki, Y. Kumamoto, A., Saito, M., \& Kaito, C. 2008, Characteristic Low-Temperature Crystallization of Amorphous Mg-bearing Silicate Grains under Electron Irradiation, ApJ, 680L, 89

Malfait, K., Bogaert, E., \& Waelkens, C. 1998, An Ultraviolet, Optical and Infrared Study of Herbig Ae/Be Stars, A\&A, 331, 211

Mannings, V. 1994, Submillimetre Observations of Herbig Ae/Be Systems, MNRAS, 271, 587

Meeus, G., Waters, L. B. F. M., Bouwman, J., van den Ancker, M. E., Waelkens, C., \& Malfait, K. 2001, ISO Spectroscopy of Circumstellar Dust in 14 Herbig Ae/Be Systems: Towards an Understanding of Dust Processing, A\&A, 365, 476

Miroshnichenko, A., Ivezić, Z., Vinković, D., \& Elitzur, M. 1999, Dust Emission from Herbig Ae/Be Stars: Evidence for Disks and Envelopes, ApJ, 520, 115L

Suh, K.-W. 1999, Optical Properties of the Silicate Dust Grains in the Envelopes around AGB Stars, MNRAS, 304, 389

Suh, K.-W. 2000, Optical Properties of the Carbon Dust Grains in the Envelopes around AGB Stars, MNRAS, 315, 740

Suh, K.-W., Kim, M.-R., \& Baek, J.-H. 2002, A Study of ISO Spectra for Herbig Ae/Be Stars, JASS, 19, 255
Tamanai, A., Mutschke, H., Blum, J., Posch, Th., Koike, C., \& Ferguson, J. W. 2009, Morphological Effects on IR Band Profiles. Experimental Spectroscopic Analysis with Application to Observed Spectra of Oxygen-Rich AGB Stars, A\&A, 501, 251

Towers, I. N., \& Robinson, G. 2009, A Model for Multiple Isothermal Circumstellar Dust Shells, Physica Scripta, 80, 015901

van den Ancker, M. E., The, P. S., Tjin A Djie, H. R. E., Catala, C., de Winter, D., Blondel, P. F. C., \& Waters, L. B. F. M. 1997, Hipparcos Data on Herbig Ae/Be Stars: an Evolutionary Scenario, A\&A, 324L, 33

Waters, L. B. F. M., \& Waelkens, C. 1998, Herbig Ae/Be Stars, ARA\&A, 36, 233

Whitney, B. A., Wood, K., Bjorkman, J. E., \& Wolff, M. J. 2003, Two-Dimensional Radiative Transfer in Protostellar Envelopes. I. Effects of Geometry on Class I Sources, ApJ, 591, 1049 